\title{
PAT for Continuous Chromatography Integrated into Continuous Manufacturing of Biologics towards Autonomous Operation
}

\author{
Florian Lukas Vetter, Steffen Zobel-Roos and Jochen Strube *
}

Citation: Vetter, F.L.; Zobel-Roos, S.; Strube, J. PAT for Continuous Chromatography Integrated into Continuous Manufacturing of Biologics towards Autonomous Operation. Processes 2021, 9, 472. https://doi.org/10.3390/pr9030472

Academic Editors: Krist V. Gernaey and Raja Ghosh

Received: 26 January 2021

Accepted: 4 March 2021

Published: 6 March 2021

Publisher's Note: MDPI stays neutral with regard to jurisdictional claims in published maps and institutional affiliations.

Copyright: (c) 2021 by the authors. Licensee MDPI, Basel, Switzerland. This article is an open access article distributed under the terms and conditions of the Creative Commons Attribution (CC BY) license (https:// creativecommons.org/licenses/by/ $4.0 /)$
Institute for Separation and Process Technology, Clausthal University of Technology, Leibnizstraße 15, 38678 Clausthal-Zellerfeld, Germany; vetter@itv.tu-clausthal.de (F.L.V.); zobel-roos@itv.tu-clausthal.de (S.Z.-R.)

* Correspondence: strube@itv.tu-clausthal.de; Tel.: +49-5323-72-2872

Abstract: This study proposes a reliable inline PAT concept for the simultaneous monitoring of different product components after chromatography. The feed for purification consisted of four main components, IgG monomer, dimer, and two lower molecular weight components of $4.4 \mathrm{kDa}$ and $1 \mathrm{kDa}$ molecular weight. The proposed measurement setup consists of a UV-VIS diode-array detector and a fluorescence detector. Applying this system, a $\mathrm{R}^{2}$ of 0.93 for the target component, a $\mathrm{R}^{2}$ of 0.67 for the dimer, a $\mathrm{R}^{2}$ of 0.91 for the first side component and a $\mathrm{R}^{2}$ of 0.93 for the second side component is achieved. Root mean square error for IgG monomer was $0.027 \mathrm{~g} / \mathrm{L}$, for dimer $0.0047 \mathrm{~g} / \mathrm{L}$, for side component $10.016 \mathrm{~g} / \mathrm{L}$ and for the side component $20.014 \mathrm{~g} / \mathrm{L}$. The proposed measurement concept tracked component concentration reliably down to $0.05 \mathrm{~g} / \mathrm{L}$. Zero-point fluctuations were kept within a standard deviation of $0.018 \mathrm{~g} / \mathrm{L}$ for samples with no IgG concentration but with side components present, allowing a reliable detection of the target component. The main reason inline concentration measurements have not been established yet, is the false-positive measurement of target components when side components are present. This problem was eliminated using the combination of fluorescence and UV-VIS data for the test system. The use of this measurement system is simulated for the test system, allowing an automatic fraction cut at $0.05 \mathrm{~g} / \mathrm{L}$. In this simulation a consistent yield of $>99 \%$ was achieved. Process disturbances for processed feed volume, feed purity and feed IgG concentration can be compensated with this setup. Compared to a timed process control, yield can be increased by up to $12.5 \%$, if unexpected process disturbances occur.

Keywords: quality by design $(\mathrm{QbD})$; process analytical technology (PAT); digital twin; chemometrics; multivariate data analysis; continuous manufacturing; real-time release testing; integrated counter current chromatography (iCCC); monoclonal antibody (mAB); Raman spectroscopy; attenuated total reflection Fourier transformed infrared spectroscopy (ATR-FTIR); fluorescence; diode array detector

\section{Introduction}

Biologic manufacturing moves toward high-yield, high-throughput continuous process alternatives as the demand for biologic pharmaceuticals appears to be steadily rising [1-4]. Furthermore, the manifold of new entities (e.g., antibody fragments, virus-like particles, and mRNA) and personalized medicines demand a more efficient production process to ensure economic success $[5,6]$. To realize this process the most viable way is the establishment of continuous chromatography, since chromatography is still the main workhorse in biopharmaceutical downstream processes $[1,2,6-8]$. While these continuous processes exist and their practicability was shown in the laboratory [1,2], a robust process analytical technology (PAT) concept has still to be established.

Most recent works focus on digital twin [9] and process control $[10,11]$ to enable continuous biomanufacturing. For breakthrough processes such as periodic counter-current chromatography (PCC) or multicolumn counter-current solvent gradient purification (MCSGP) [12,13], UV-VIS sum signal detection has long been state of the art as a switch 
criteria $[14,15]$. For other chromatographic processes, like standard batch chromatography or integrated counter-current chromatography (iCCC), an inline measurement of multiple components (e.g., the main component and side components) would be promising to simplify process control. Most continuous processes do not need a rapid inline measurement since changes occur over long times. In chromatography, on the other hand, a fast, inline measurement is urgently needed. As the inline detection of side components during elution can be used to fine-tune the switch criteria, which majorly impact process yield and purity. UV-VIS spectra analysis and peak deconvolution has proven its potential as an inline target component measurement $[11,16]$. Other measurement methods have been used to enable inline concentration measurement. Raman was successfully employed for breakthrough detection of a single component [17], FTIR has also been successfully employed to determine target component concentration or secondary structure integrity [18-20]. For the measurement of fibroblast growth factor 2 from Escherichia coli, fluorescence measurements were successfully employed to measure the target component and host cell protein concentrations using a single partial-least squares (PLS) model [21]. While yielding good results in model mixtures and single-component solutions, signal overlapping can be a sizable problem for a PAT- and chemometrics-based process control [22].

The aim of this study is to test UV-VIS, FTIR, fluorescence, and Raman spectroscopy, to propose a method for advanced process control (APC) of continuous chromatography, using integrated counter-current chromatography as an example [23]. To achieve this, firstly single detectors are tested on their feasibility for component measurement in the chromatographic product. Secondly, combinations of these detectors are tested and a proposal of the best method is given. Thirdly, the proposed APC system is simulated to show the proposed usage and the handling of potential incidents.

\section{Materials and Methods}

\subsection{Experimental Setup}

An antibody solution obtained after precipitation was used in the chromatography studies. Further description about cultivation, liquid-liquid extraction and precipitation can be taken from the overview paper [24]. Purification was accomplished using a YMC S75 strong cation-exchange column (YMC-BioPro S75, $26 \times 7.0 \mathrm{~mm}$ ID, YMC Co., Ltd., Kyoto, Japan). Five column volumes (CV) were loaded onto the column. For flow-through-FTIR and Raman $30 \mathrm{CV}$ were loaded on the column. After loading, the column is washed with $3 \mathrm{CV}$ of $50 \mathrm{mM}$ sodium phosphate buffer at $\mathrm{pH}=5,5$ (disodium phosphate dihydrate, EMSURE $^{\circledR}$, Merck KgaA, Darmstadt, Germany; anhydrous sodium dihydrogen phosphate, EMSURE $^{\circledR}$, Merck KgaA, Darmstadt, Germany). After washing the product was obtained by a gradient elution using the same buffer modified with $1 \mathrm{M}$ sodium chloride (EMSURE ${ }^{\circledR}$, Merck KgaA, Darmstadt, Germany). Varying gradient lengths of 5, 10 and 15 CV were employed. After elution the column was regenerated with $5 \mathrm{CV}$ and equilibrated with $3 \mathrm{CV}$. The eluted product was fractioned into $30 \mathrm{~s}$ steps. Samples of $1 \mathrm{~mL}$ were obtained in two repetitions.

The chromatographic setup consisted of a VWR-Hitachi LaChrom Elite ${ }^{\circledR}$ system $\left(\mathrm{VWR}^{\circledR}\right.$, Darmstadt, Germany). It was equipped with a quaternary gradient pump (L-2130), an Autosampler (L-2200) and a diode array detector (L-2455). Between the chromatographic setup and the fraction collector (Foxy Jr. ${ }^{\circledR}$, Teledyne Isco, Lincoln, NE, USA) the following inline analytics were implemented. For UV-VIS spectra measurements a diode-arraydetector (Smartline DAD 2600, Knauer Wissenschaftliche Geräte GmbH, Berlin, Germany) was installed. For FTIR-Spectra a benchtop FTIR Detector (Alpha II, Bruker Corporation, Billerica, MA, USA) was equipped with a flow-through cell. Raman spectra were measured using a Raman spectrometer (Diode laser, 785 nm, Ocean Optics BV, Ostfildern, Germany) in conjunction with a flow-through cell (Durchfluß-Küvette 176.700, Hellma GmbH and Co., KG, Müllheim, Germany). Fluorescence emission spectra were measured at-line in the obtained fractions using a fluorescence detector (Jasco FP-2020, Jasco Deutschland GmbH, Pfungstadt, Germany). 


\subsection{Identification of Components}

Proteins eluting from the column can be divided into four groups. The chromatogram resulting from preperative ion-exchange chromatography (IEX) and the corresponding size-exclusion chromatography of three representative obtained fractions is depicted in Figure 1. The first group elutes after $12 \mathrm{~min}$ in IEX. Evaluating the SEC, see Figure 1b, the first side component group consists of a light molecular weight component (LMW) at $4.4 \mathrm{kDa}$. The second group elutes after $15.5 \mathrm{~min}$ in IEX, and consist of a side component at $1 \mathrm{kDa}$ molecular weight. The first side component is further on denoted as LMW 1 and the second as LMW 2. These components are pseudo-components; for their evaluation the peak area between 10 and 13 min for LMW 1 and the area between 13 and 15 min for LMW 2 were added.

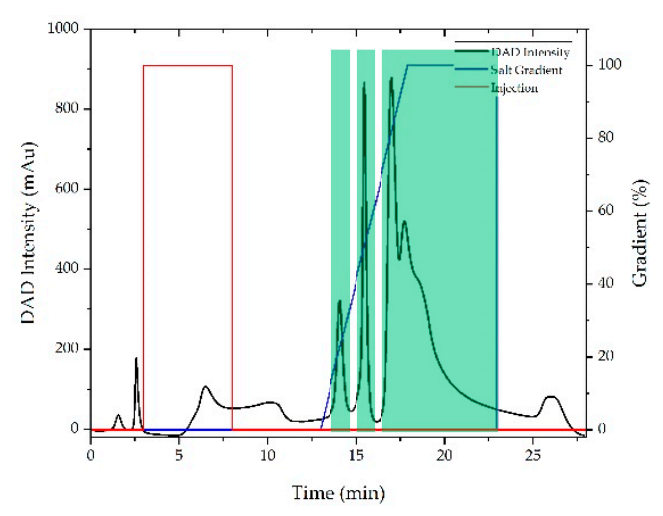

(a)

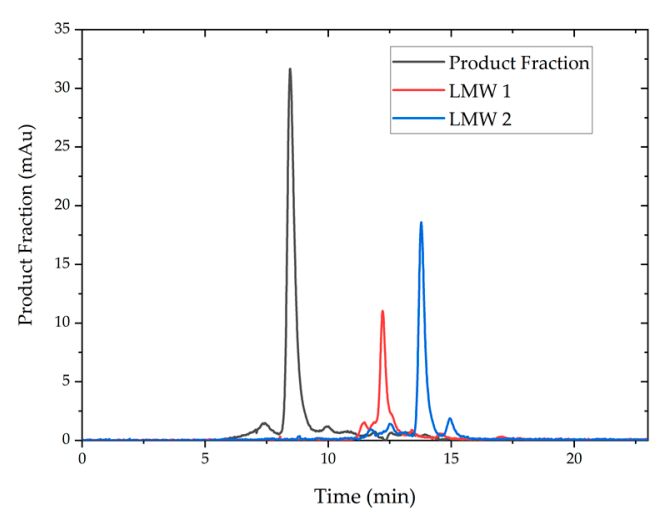

(b)

Figure 1. IEX Chromatogram of a $10 \mathrm{CV}$ salt gradient (a), marked in green are the different eluent fraction. On the right $(\mathbf{b})$ a representative SEC chromatogram of each eluent fraction is shown.

The IgG monomer and dimer elute in IEX after $16 \mathrm{~min}$. They elute in the same fraction and are identified using SEC and Protein A affinity chromatography. The retention times in SEC are compared to a polyclonal antibody solution (Gammanorm, Octapharma AG, Lachen, Switzerland) to identify monomer and dimer of the IgG.

\subsection{Data Analysis}

For online measured data the spectra obtained in a chromatographic fraction were averaged. This was done using the DAD of the Elite LaChrom ${ }^{\circledR}$ system, the chromatogram provided by the Smartline DAD and the system dead volume for temporal allocation of the spectroscopic online data to the correct fraction.

Averaging and reduction of spectra was done, using Spectragryph (Dr. Friedrich Menges Software-Entwicklung, Oberstdorf, Germany). Raman spectra were shortened to $1800-400 \mathrm{~cm}^{-1}$. FTIR spectra were shortened to $1800-800 \mathrm{~cm}^{-1}$. Fluorescence emission spectra were shortened to 580-700 nm, DAD spectra were shortened to $200-300 \mathrm{~nm}$.

All spectra were pre-processed, using the methodology laid out in the overview paper of this study, to reduce baseline drift or scatter effects identified in the scatter effects plot [24]. The application of this methodology led to a standard normal variate standardization (SNV) for FTIR and a 1st order derivative for the Raman data. Fluorescence and UV-VIS spectra were not further pre-processed. Pre-processing and PLS modelling were done using the data analysis software Unscrambler X (Camo Analytics, Oslo, Norway). PLS models were built for the four different analytes: target component, IgG dimer, low molecular weight components 1 , and low molecular weight components 2 . The peak areas of these analytes obtained from the SEC after purification on IEX were used as the y-matrices for PLS regression. The pre-processed and shortened spectra were used as the $\mathrm{x}$-matrices. The data set for model building consisted of three experiments, of gradients 5, 10, $15 \mathrm{CV}$ and two repetitions each. FTIR and Raman PLS models were cross-validated. For cross-validation, 
a leave one out cross-validation (LOO-CV) was used. DAD and fluorescence PLS models were validated using another, independent experiment.

\subsection{Combination of Spectroscopic Data}

$\mathrm{DAD}$ and fluorescence spectroscopic data were combined, since they have shown to be sensitive for different side components. DAD showed a better prediction for LMW 1, fluorescence for LMW 2. For combination four experiments at different gradient slopes have been combined. Since DAD and fluorescence data differ by magnitudes, a z-score standardization was performed using the arithmetic mean and standard deviation of each data set. The PLS model for the combination of spectra was validated using cross-validation. For this validation also leave one out cross-validation was used.

\subsection{Online Analytics}

Data acquisition in Raman spectroscopy was set to automatically average a total of three spectra, each of which had $1 \mathrm{~s}$ of integration time. For each sample at least 10 spectra were measured. Raman spectra were obtained from $4000-400 \mathrm{~cm}^{-1}$. Data acquisition in FTIR spectroscopy was set to automatically average a total of eight spectra, each of which had $1 \mathrm{~s}$ of integration time. FTIR spectra were obtained from $4000-400 \mathrm{~cm}^{-1}$. Data acquisition in UV-VIS spectroscopy was set to continuously record at a sampling rate of $0.2 \mathrm{~s}$, each of which had $32 \mathrm{msec}$ of integration time. DAD spectra were obtained from 190-520 $\mathrm{nm}$. Inline measurement using the fluorescence detector was not possible, since a spectra measurement takes about $30 \mathrm{~s}$. Hence, fluorescence was measured at-line. Gain was set to 1 or 10, depending on the concentration. Attenuation was set to 256 . Fluorescence measurement were done using the $16 \mu \mathrm{L}$ flow-cell, provided with the detector. The emission spectra were measured using an excitation wavelength of $280 \mathrm{~nm}$. Spectra were obtained from $280-900 \mathrm{~nm}$.

\subsection{Offline}

Immunoglobulin G concentration was measured using analytical Protein A chromatography. Side component concentrations and purity were measured using size exclusion chromatography. Protein A chromatography was performed using PA ID Poros ${ }^{\circledR}$ Protein A Sensor Cartridges (Applied Biosystems, Waltham, MA, USA). For Protein A, Dulbecco's PBS buffer (Sigma-Aldrich, St. Louis, MO, USA) was used as loading buffer at $\mathrm{pH} 7.4$ for elution the PBS buffer was used at $\mathrm{pH}$ 2.6. For size-exclusion chromatography a Yarra ${ }^{\circledR}$ SEC-3000 column (Phenomenex ${ }^{\circledR}$ Inc., Torrance, CA, USA) was employed as the buffer system a PBS buffer with $0.1 \mathrm{M} \mathrm{Na}_{2} \mathrm{SO}_{4}, 0.1 \mathrm{M} \mathrm{Na}_{2} \mathrm{HPO}_{4}$ and $0.1 \mathrm{NaH}_{2} \mathrm{PO}_{4}$ (Merck KGaA, Darmstadt, Germany) was used. The evaluated chromatogram was measured at $280 \mathrm{~nm}$.

Biological activity, overall protein concentration and side component analysis were monitored using ELISA, Bradford, SDS-page and DNA-assays. The methodology and results can be checked in the overview paper of this study [24].

\subsection{Simulation}

To show the viability and effect of a chromatographic process being controlled via an inline concentration measurement, the process is simulated using a concentration-based fractionation of the product and a timed fractionation.

Simulation of chromatography is usually done using either a general rate model or a lumped pore diffusion model $[10,25,26]$. In biochromatography, due to the large molecular size of the biomolecules, the general rate model shows the closest relation to the real process, as pore diffusion is modelled. However, to minimize calculation times in the model-based control of chromatography. The linearization of the pore diffusion significantly lowers the required calculation times and thereby allows for shortened calculation times to enable process control [27]. In this case study a lumped pore diffusion model of chromatography is used. This leads to a reduction in simulation time, making iCCC simulations significantly 
faster. Mass balance of the stationary phase for the general rate model (Equation (1)) and the lumped pore diffusion model (Equation (2)) are [25]:

$$
\begin{gathered}
\varepsilon_{p, i} \cdot \frac{\delta c_{p, i}}{\delta t}+\left(1-\varepsilon_{p, i}\right) \cdot \frac{\delta q_{i}}{\delta t}=\frac{1}{r^{2}} \frac{\delta}{\delta r}\left[r^{2}\left(\varepsilon_{p, i} \cdot D_{p, i} \cdot \frac{\delta c_{p, i}}{\delta r}+\left(1-\varepsilon_{p, i}\right) \cdot D_{S, i} \frac{\delta q_{i}^{*}}{\delta r}\right)\right] \\
\varepsilon_{p, i} \cdot \frac{\delta c_{p, i}}{\delta t}+\left(1-\varepsilon_{p, i}\right) \cdot \frac{\delta q_{i}}{\delta t}=\frac{6}{d_{P}} \cdot \frac{\left(1-\varepsilon_{S}\right)}{\varepsilon_{S}} \cdot k_{e f f, i} \cdot\left(c_{i}-c_{p, i}\right)
\end{gathered}
$$

With $\varepsilon_{P, i}$ as porosity of the component, $c_{p, i}$ as concentration of the component in the pores, $t$ as time, $q_{i}$ as loading, $d_{P}$ as mean diameter of the resin particle, $\varepsilon_{S}$ as voidage, $k_{e f f, i}$ as the effective mass transport coefficient and $c_{i}$ as concentration in the continuous phase.

Different approaches for modelling of adsorption have been described by different working groups $[25,26,28-30]$. In this study adsorption is modelled using a Langmuir isotherm [29,31], see Equation (3). In previous studies and studies of different working groups Langmuir has shown an accurate description of the adsorption of biomolecules in chromatography $[10,26,32]$.

$$
q_{i}=\frac{q_{\max , i} \cdot K_{e q, i} \cdot c_{i}}{1+K_{e q, i} \cdot c_{i}}
$$

Here, $q_{\max , i}$ is the maximum loading capacity of the component and $K_{e q, i}$ is the Langmuir coefficient of the component. $K_{e q, i}$ and $q_{\max , i}$ are related by the Henry coefficient $H_{i}$, see Equation (4) [25]. Salt influence can be described by Equation (5) and Equation (6) defining $a_{1}, a_{2}, b_{1}$ and $b_{2}$ as correlation coefficients [26,33].

$$
\begin{gathered}
q_{\max , i} \cdot K_{e q, i}=H_{i} \\
q_{\max , i}=b_{1} \cdot c_{p, 1}+b_{2} \\
H_{i}=a_{1} \cdot c_{p, 1} a_{2}
\end{gathered}
$$

The mass transfer coefficient $k_{e f f, i}$ is given by Equation (7). Here, $k_{f, i}$ is the film mass transfer coefficient, $r_{p}$ the particle radius and $D_{p, i}$ the pore diffusion coefficient.

$$
k_{e f f, i}=\frac{1}{\frac{1}{k_{f, i}}+\frac{r_{p}}{D_{p, i}}}
$$

$D_{p, i}$ is calculated according to the correlation of Carta [34] and $k_{f, i}$ according to Wilson and Geanoplis [35].

\subsubsection{Parameter Determination}

Based on previous works parameter determination was split in three parts [26]. Firstly, the fluid dynamic parameters were given by the resin supplier, e.g., the particle size, pore size or porosity. The kinetic parameters $\left(D_{p, i}\right.$ and $\left.k_{f, i}\right)$ were correlated and the particle torosity was taken from earlier works [10]. Thirdly, the isotherm parameters to demonstrate potential process control using a digital twin were determined. The determination of the isotherm parameters was based on the chromatograms obtained in the experiments described above. The determination concept of the isotherm parameters is briefly depicted in Figure 2. Comparing the simulation results and the experimental data, firstly the simulated retention time is fitted to experimental data. The main influence on retention time is $a_{1}$ and $a_{2}$ as these parameters determine the Henry coefficient. Then the peak form is fitted to the experimental data adjusting $b_{1}$ and $b_{2}$. After the experimental data is sufficiently described by the simulation, the mentioned parameters $\left(a_{1}, a_{2}, b_{1}\right.$ and $\left.b_{2}\right)$ are fine-tuned using a Nelder-Mead estimation routine, based on the start values obtained in the first two steps. The parameters were optimized for least square errors between the experimental and measured data. 


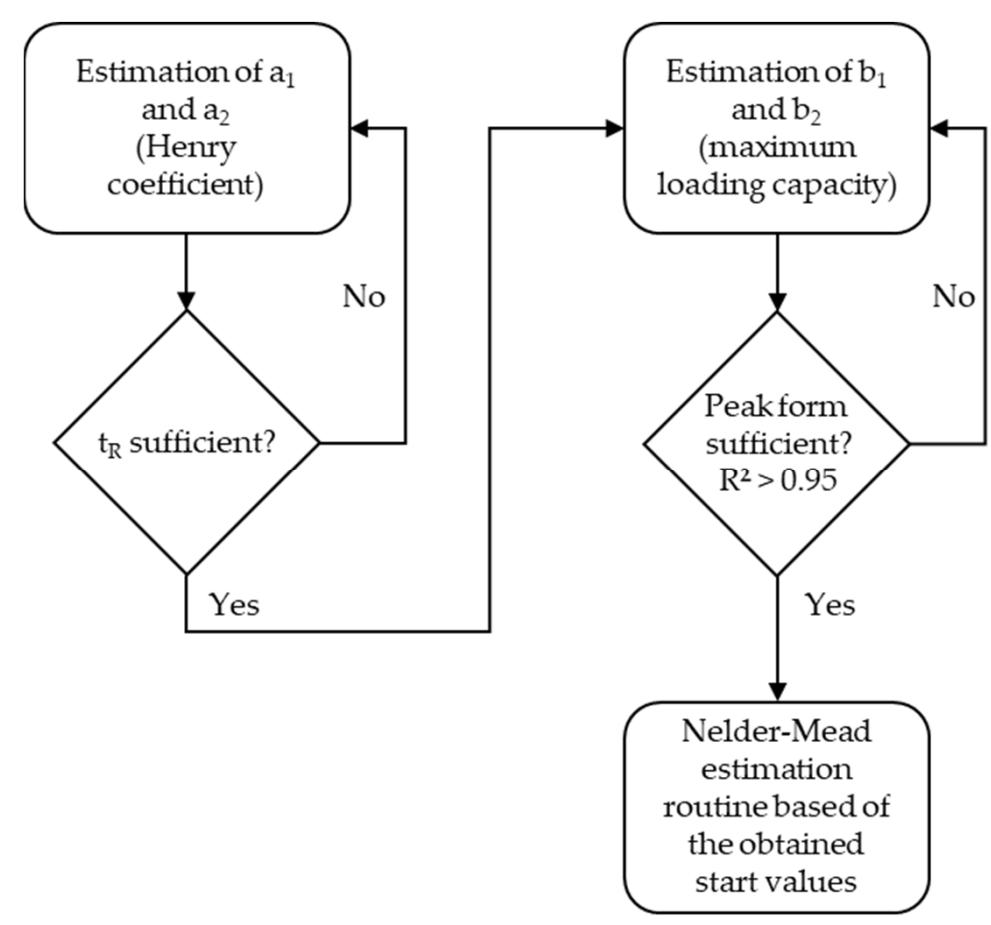

Figure 2. Determination concept of isotherm parameters.

\subsubsection{Process Model Overview}

The overall process model consists of two chromatography columns, with modelled splitting arrays based on the iCCC process explained previously [23]. The product split is done automatically in the model, based on product concentration. For simulation the studies, product fraction was cut starting at $0.05 \mathrm{~g} / \mathrm{L} \mathrm{iGg}$ concentration until concentration reaches $0.05 \mathrm{~g} / \mathrm{L}$ again in IEX. HIC fractions were cut accordingly. Since in iCCC a recycled fraction from HIC is loaded to the IEX column in the following cycle a higher salt concentration fraction is loaded onto the ion exchange column. This increase in salt concentration my lead to involuntarily product elution. To avoid this, loading was adjusted to $50 \%$ of the dynamic binding capacity to avoid product elution during loading of this fraction.

\section{Results of PAT}

\subsection{Scatter Effects}

For the evaluation of scatter effects observed in Raman spectroscopy, a preprocessing method is chosen. In Figure 3a un-preprocessed spectral data is shown. These show a $y$-axis drift, and therefor additive effects. To eliminate these, 1st derivatization of spectral data is chosen to preprocess the spectral data. Prior to derivatization the spectra were smoothed applying a Savitzky-Golay filter in a 11-point symmetric kernel with a 2nd order polynomial. The preprocessed data is shown in (b). Since no additional effects were observed, the data was not further preprocessed.

In Figure 4 the course of the FTIR spectral data is depicted. Here the lowest IgG concentration is colored red and the highest blue. For preprocessing, the scatter effect plots show different slopes. This points to scatter effects; hence a normalization was performed using the Standard Normal Variate (SNV) method provided in the data analysis software. Un-preprocessed data is shown in Figure $4 a$. Figure 4 b shows pre-processed data. Further preprocessing was not employed, as no additive effects can be observed. 


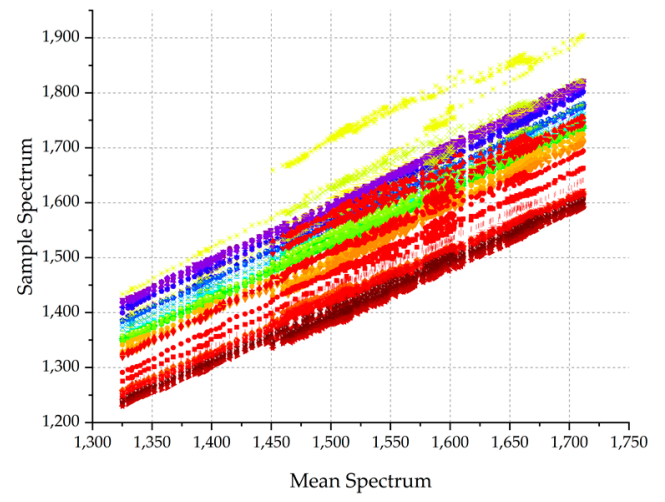

(a)

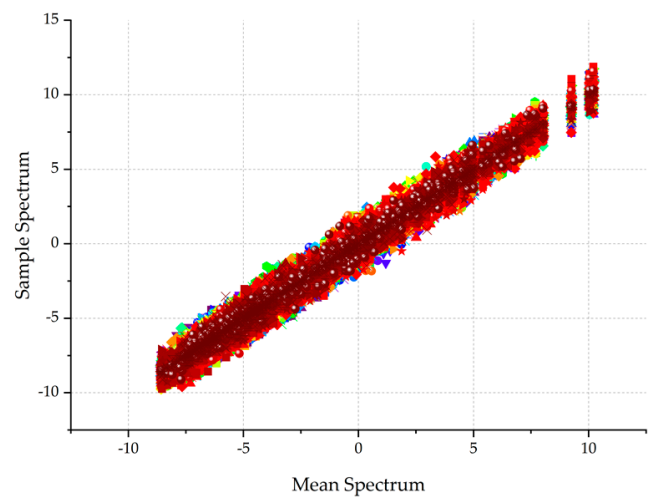

(b)

Figure 3. Raman scatter effects plots for evaluation of needed preprocessing of spectral data (a) shows scatter effects of raw spectral data and (b) shows scatter effects after preprocessing.

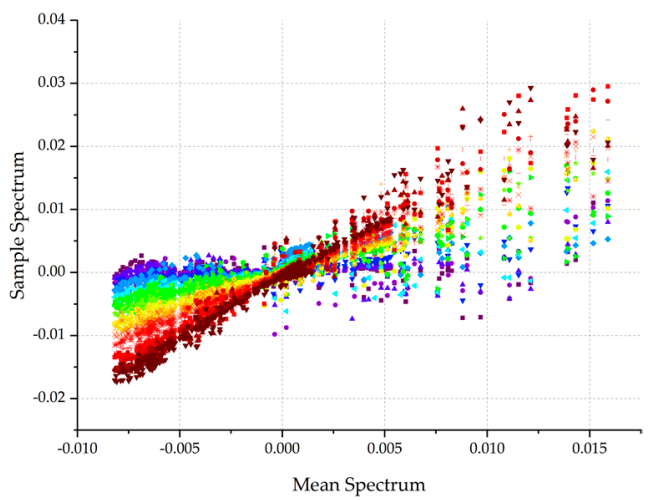

(a)

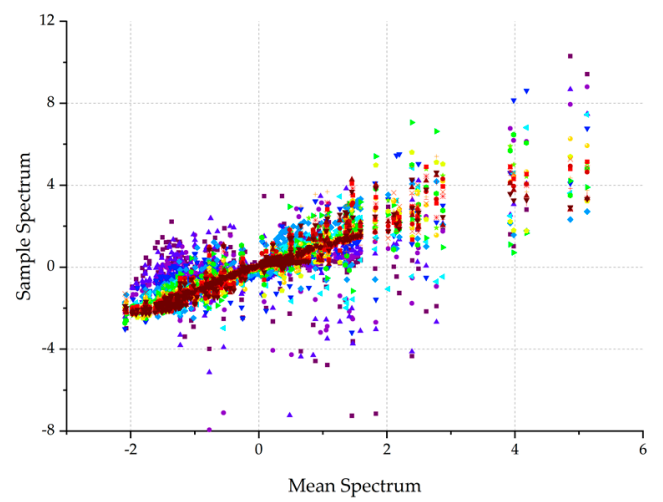

(b)

Figure 4. FTIR scatter effect plot for chromatography, (a) show scatter effects of raw spectral data and (b) show scatter effects after preprocessing.

DAD spectra ranges were shortened to $200-300 \mathrm{~nm}$, as this is the only part of the measured spectra, were significant absorption can be observed. In Figure 5, the scatter plot for DAD data is illustrated. The spectral data show complex effects. This is due to the fact, that the effects are caused by the identified components and no underlying effects are present. Hence any preprocessing significantly worsened PLS regression, eliminating effects the PLS model can explain with the given concentrations.

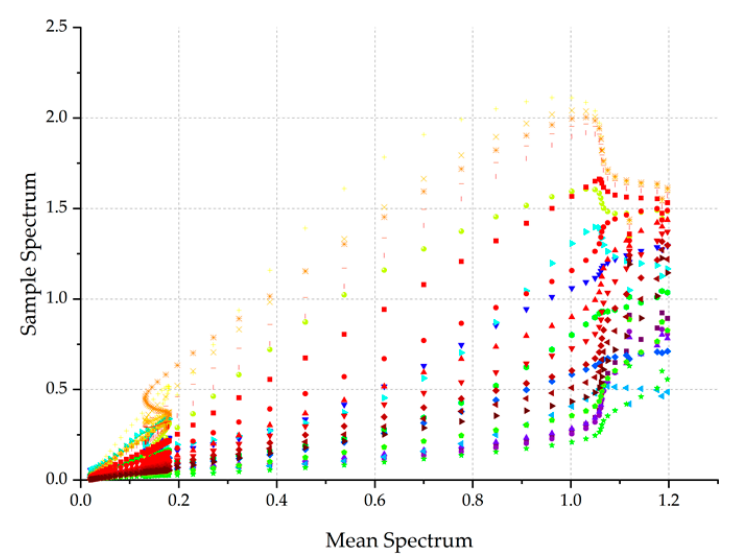

Figure 5. DAD scatter effects plot for un-preprocessed spectral data, obtained in chromatography. 
Fluorescence spectra width was shortened to 580-700 nm. In Figure 6 the scatter effects of fluorescence data are illustrated. As in DAD spectroscopy these spectra show a complex behavior, and as well as in the DAD chromatography experiment, the observed effects can be explained using the given concentrations. Fluorescence data was also not preprocessed, which would eliminate some of the information contained in the spectra, negatively impacting PLS regression.

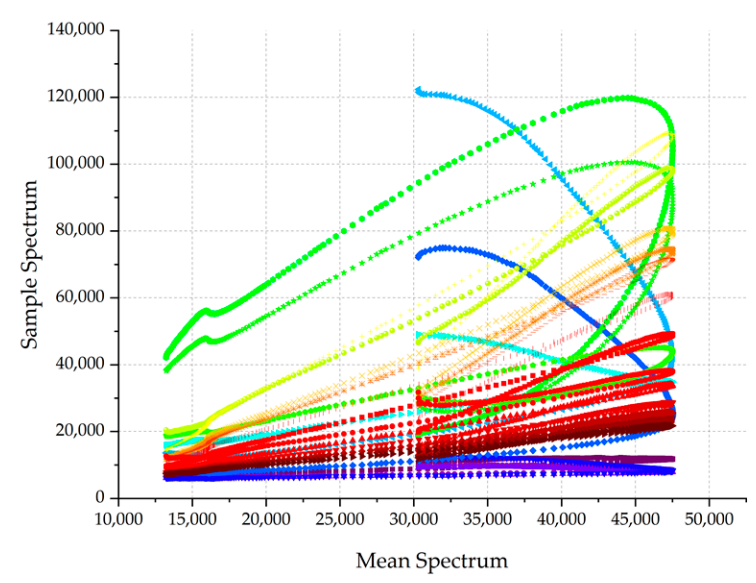

Figure 6. Scatter effects plot for fluorescence spectral data obtained in chromatography.

\subsection{PLS Models}

PLS validation for Raman spectroscopy in chromatography was not possible, as portrayed in Figure 7c. The trained PLS showed a $R^{2}$ of 0.968 , for the validation set the $R^{2}$ was -0.52 . The reason for this is that the large flow-cell volume in combination with the low flow rate results in a long retention time of the product inside the cell and therefore favors back-mixing. This is further discussed in the overview paper [24].

Raw Data

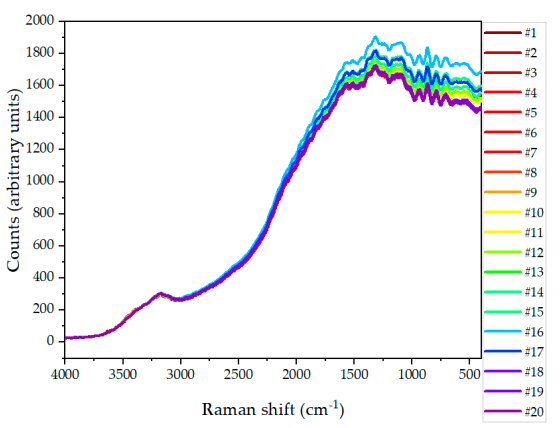

(a)
Pre-processed

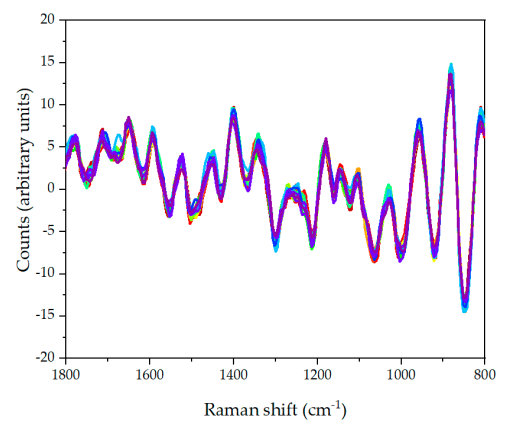

(b)

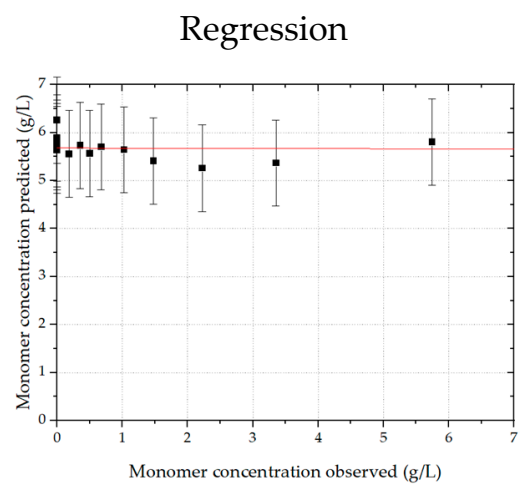

(c)

Figure 7. Data of Raman spectra (a), pre-processed spectra (b) and PLS results (c). Spectra are color-coded from high concentration (blue) to low concentration (red).

Using the FTIR data, the PLS modelling for IgG resulted in a $\mathrm{R}^{2}$ of 0.92 including validation samples. The RMSE was $0.037 \mathrm{~g} / \mathrm{L}$. In Figure $8 \mathrm{c}$ the regression results are shown. In comparison to DAD and fluorescence the salt gradient shows a strong influence on FTIR spectra. This is especially apparent between the wavenumbers $1700-1600 \mathrm{~cm}^{-1}$ and $1000-800 \mathrm{~cm}^{-1}$. The drift between 1000 and $800 \mathrm{~cm}^{-1}$ results mainly from the higher salt concentration, as there is a shift from lower salt concentrations (blue) to higher salt concentrations (red), see Figure $8 \mathrm{~b}$. Between 1700 and $1600 \mathrm{~cm}^{-1}$ the increase is mainly due to higher IgG concentration, as the high salt concentrations do not contribute to a rise in intensity. Best fit was achieved using three factors in the PLS model. 
Raw Data

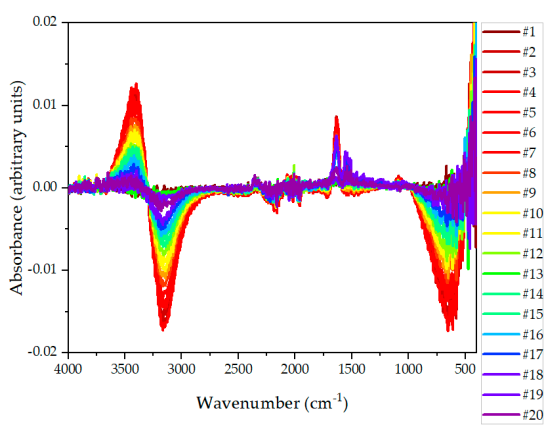

(a)

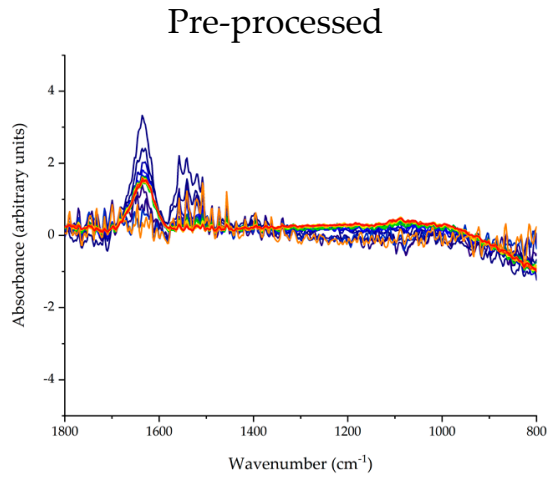

(b)

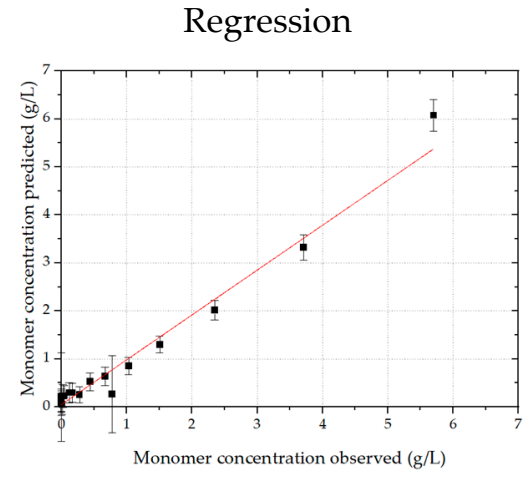

(c)

Figure 8. Data of FTIR spectra (a), pre-processed spectra (b) and PLS results (c). Spectra are color-coded from high concentration (blue) to low concentration (red).

For the shortened DAD spectra, a $\mathrm{R}^{2}$ of 0.94 and a RMSE of validation of $0.022 \mathrm{~g} / \mathrm{L}$ for the IgG monomer was achieved in chromatography. The regression results are illustrated in Figure 9. In the regression plot (c) a high variance for the zero-concentration samples is shown. This results from the similar spectra (b). Between 200 and $225 \mathrm{~nm}$ the side components show a strong absorbance, like the IgG monomer. The component specificity of this model results from the stronger absorbance of IgG around $275 \mathrm{~nm}$. While the PLS model can eliminate these overlapping effects to some degree, it is not possible to eliminate this overlapping completely. Best fit was achieved using six factors in the PLS model.

Raw Data

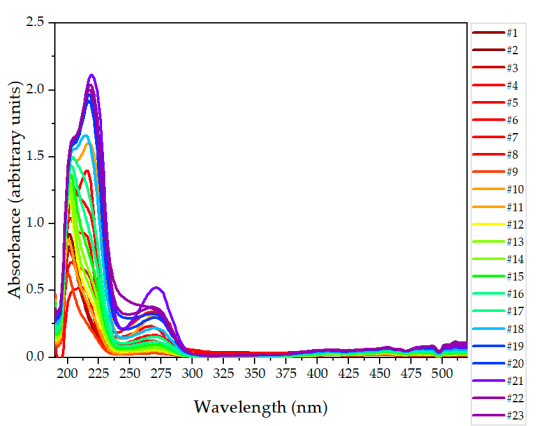

(a)

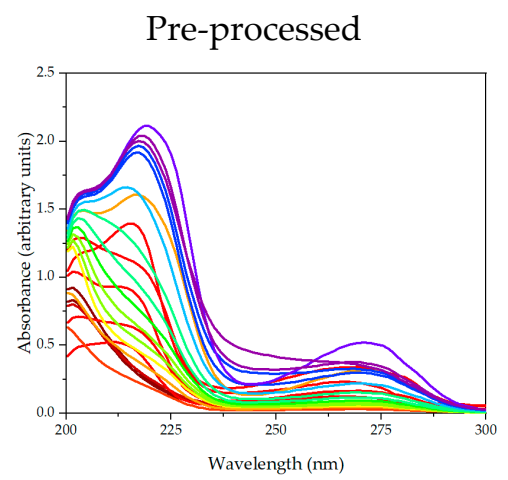

(b)

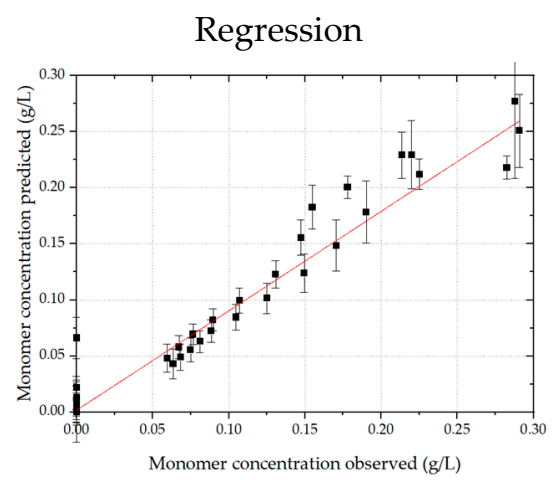

(c)

Figure 9. Data of DAD spectra (a), shortened spectra (b) and PLS results (c). Spectra are color-coded from high concentration (blue) to low concentration (red).

For fluorescence the $\mathrm{R}^{2}$ was 0.93 the RMSE of validation was $0.022 \mathrm{~g} / \mathrm{L}$. The regression results are depicted in Figure 10. In comparison to DAD the fluorescence model predicts low concentration samples more accurately. This can be explained interpreting the spectra, see Figure 10b. The samples containing high concentrations of IgG emit most light at around $660 \mathrm{~nm}$, while for low IgG concentration samples, and high side concentration samples the fluorescence maximum is shifted to $680 \mathrm{~nm}$ and $600 \mathrm{~nm}$, respectively. 


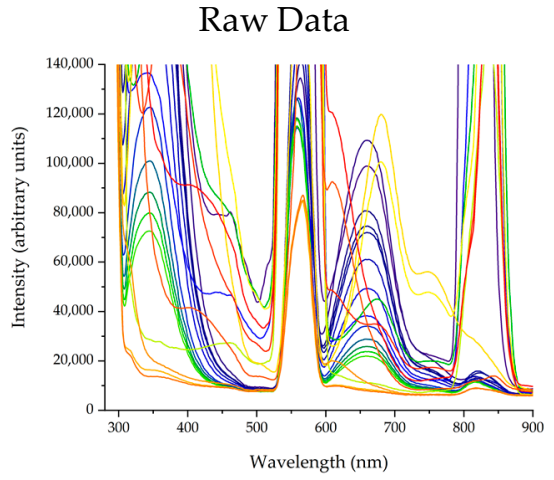

(a)
Pre-processed

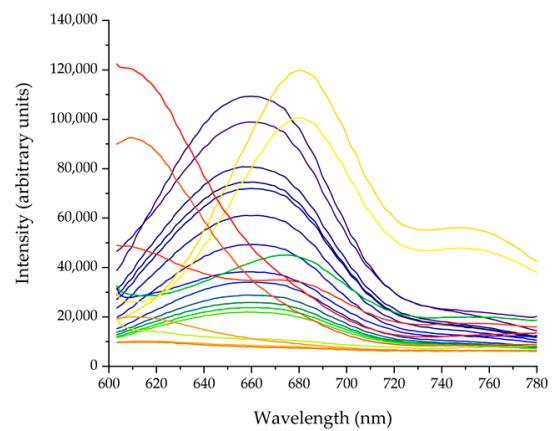

(b)
Regression

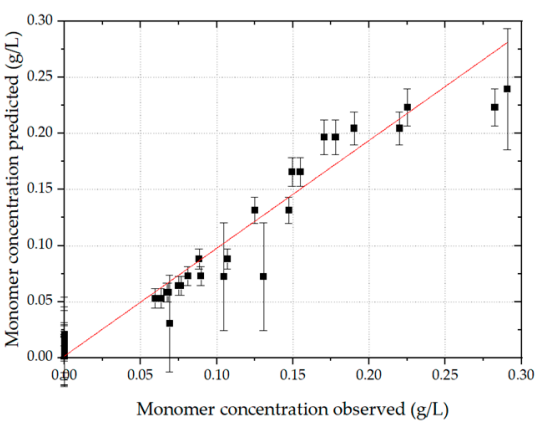

(c)

Figure 10. Data of fluorescence spectra (a), shortened spectra (b) and PLS results (c). Spectra are color-coded from high concentration (blue) to low concentration (red).

\subsection{Combination of Spectroscopic Data}

Combination of DAD and fluorescence data, showed significant improvement in prediction quality. The regression results are given in Figure 11.

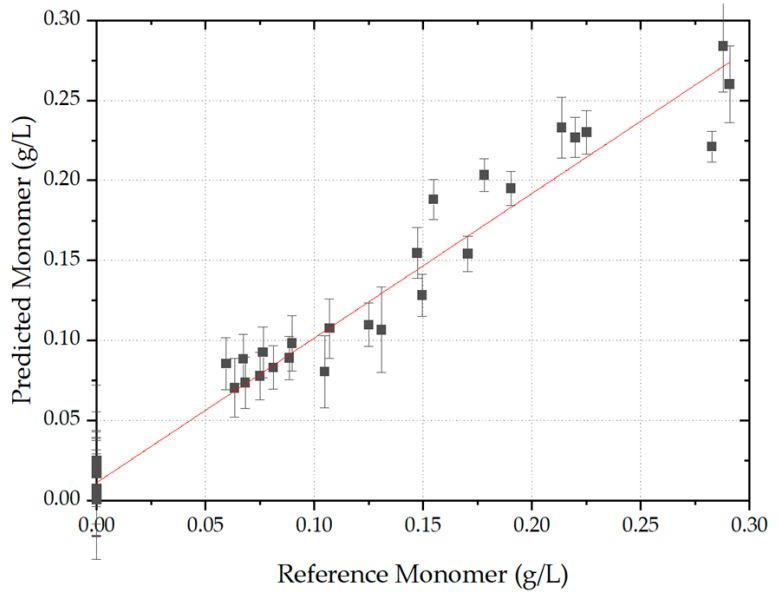

(a)

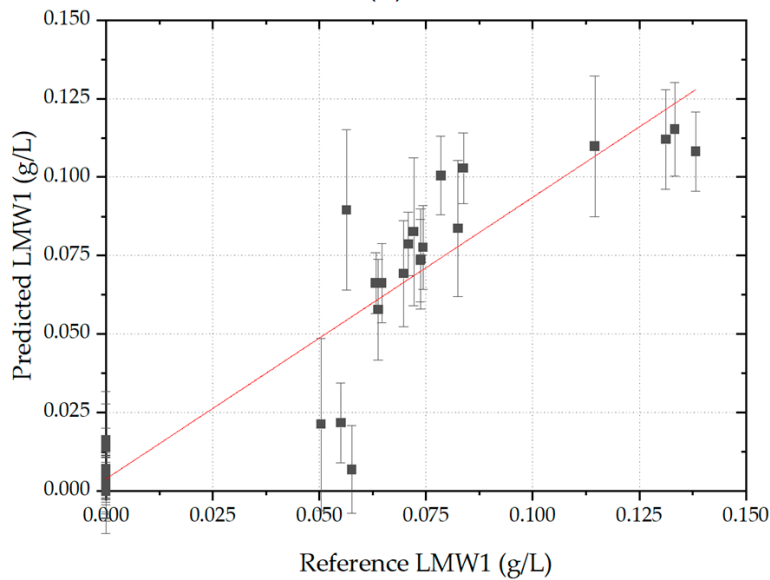

(c)

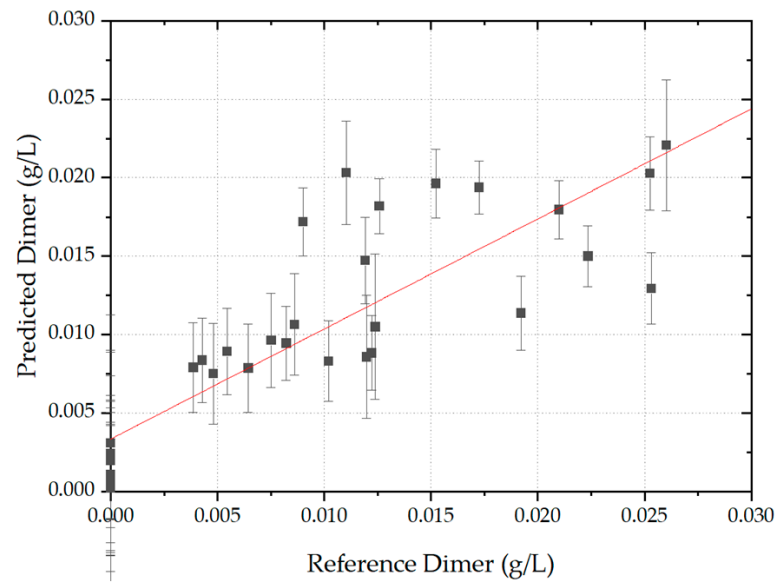

(b)

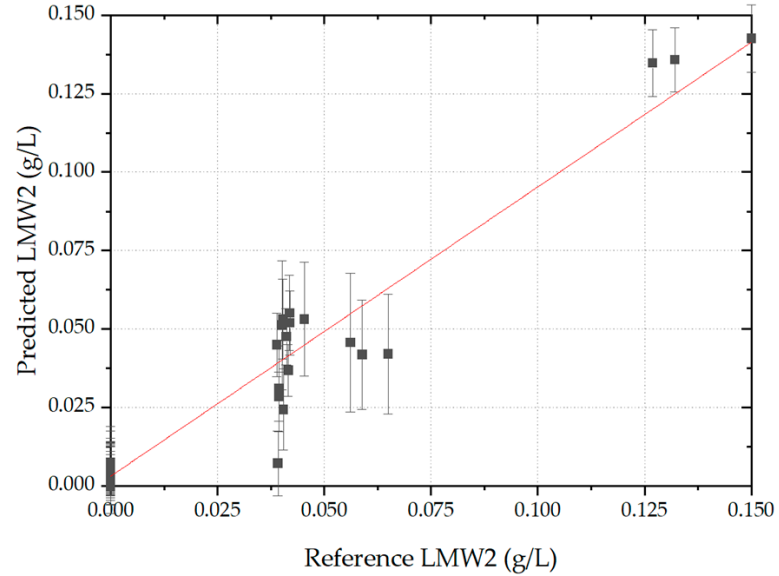

(d)

Figure 11. Prediction of process data using combination of DAD and fluorescence. IgG concentration (a), Dimer concentration (b), LMW 1 (c), LMW 2 (d). 
In Figure 12 regression coefficients for DAD and fluorescence are shown. Here, the content-specific regions in the observed spectra can be identified. LMW 2 correlates largely to the wavelengths between 190 and $210 \mathrm{~nm}$ in DAD. This already became noticeable in DAD evaluation. LMW 1 is largely correlated in DAD between 210 and $220 \mathrm{~nm}$ and fluorescence between 600 and $620 \mathrm{~nm}$. Specificity between LMW 1 and 2 results mostly from the difference at around $200 \mathrm{~nm}$ in DAD and from the shifted $x$-axis intersection point in fluorescence at around $670 \mathrm{~nm}$. Distinction between IgG monomer and dimer is observed at around $210 \mathrm{~nm}$ in the DAD.

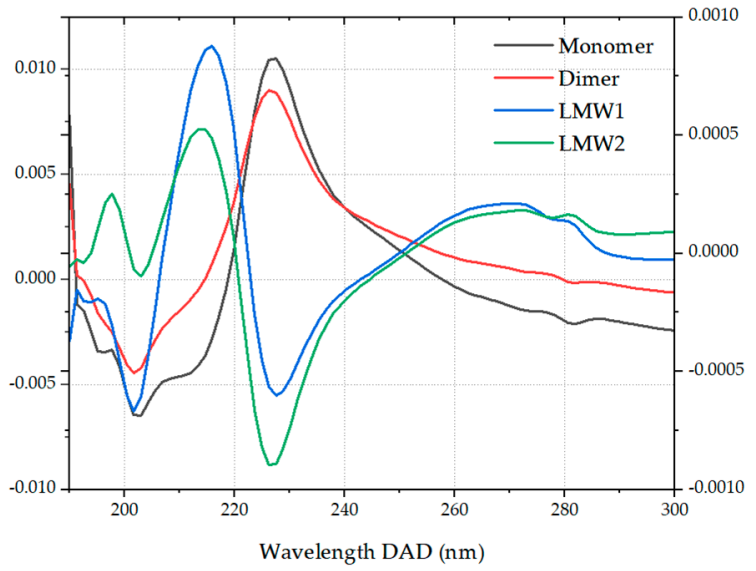

(a)

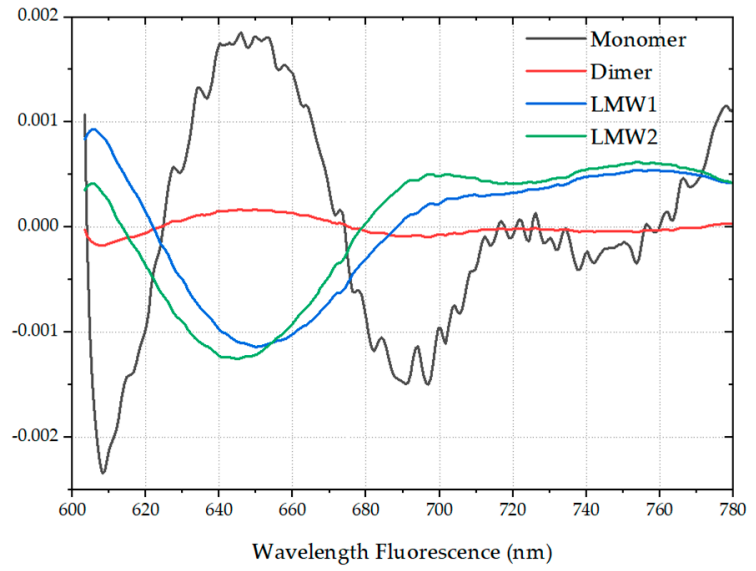

(b)

Figure 12. Regression coefficients for DAD (a) and fluorescence (b) for correlated wavelengths.

In Table 1 different coefficients of determination for the combination model, DAD model and fluorescence model are given. Regarding the determination of LMW 1 and LMW 2 , coefficients are improved using the combined model. It turns out, that a combination does not result in the best regression being used, but that the information of the DAD and fluorescence spectrum complement each other.

Table 1. Coefficient of determination $\left(R^{2}\right)$ for different product contents and PLS models.

\begin{tabular}{ccccc}
\hline Spectra & Monomer $\mathbf{R}^{\mathbf{2}}$ & Dimer $^{\mathbf{2}}$ & LMW 1 $^{\mathbf{2}}$ & LMW 2 $^{\mathbf{2}}$ \\
\hline DAD & 0.94 & 0.61 & 0.76 & 0.17 \\
Fluorescence & 0.93 & 0.84 & 0.67 & 0.90 \\
Combination & 0.93 & 0.67 & 0.91 & 0.93 \\
\hline
\end{tabular}

In Table 2 the root-mean-square error (RMSE) for different components are given. These can be understood, as the standard error resulting from the concentration measurements via PLS. There should be noted, that this also includes the error resulting from analytical chromatography and any errors in time allocation, as the PLS is based on these measurements.

Table 2. Root-mean-square error of validation (RMSE) for different product contents and PLS models.

\begin{tabular}{|c|c|c|c|c|}
\hline Spectra & Monomer RMSE/g $\cdot \mathrm{L}^{-1}$ & Dimer RMSE/g. $\mathrm{L}^{-1}$ & LMW 1 RMSE $/ g \cdot L^{-1}$ & LMW 2 RMSE/g. L ${ }^{-1}$ \\
\hline DAD & 0.023 & 0.0073 & 0.022 & 0.037 \\
\hline Fluorescence & 0.022 & 0.0034 & 0.022 & 0.013 \\
\hline Combination & 0.027 & 0.0047 & 0.016 & 0.014 \\
\hline
\end{tabular}


The RMSE has a similar course as the coefficients for determination, where there is a low determination coefficient, there is also a high RMSE observed. This is mostly to be expected, and it should be noted that for the combination the RMSE is consistently low. The RMSE results in a 5 to $10 \%$ relative error for the high concentration samples.

In addition, the low prediction error for spectroscopic data with no IgG content, which was observed in fluorescence data but not in DAD data, was reproduced, see Table 3. Here, the arithmetic mean and standard deviation of the samples containing no IgG for the different spectroscopic methods are given. The arithmetic mean of these zero-point measurement is closest to zero using the combination of DAD and fluorescence. Which means that this measurement is the most accurate. The most precise measurement seems to be fluorescence as the standard deviation of the observed data is lowest. DAD overall is a very good method to measure the target component concentration, as it is the second in accuracy for high concentration samples, see Table 2 . However, the problem with DAD is, that the samples show outliers, especially samples where there is no IgG present. The maximal deviation from zero was 0.066 and therefor the highest of the three discussed. Using this detection method, the limit of detection might be significantly lower. For FTIR the zero-point measurements showed the worst results, which was also the reason why this experiment had to be repeated with higher concentrations. Here the highest deviation from the zero point was $0.204 \mathrm{~g} / \mathrm{L}$.

Table 3. Average, standard deviation, minimal and maximal measurement of zero-point measurements.

\begin{tabular}{ccccc}
\hline Method & Average/g $\cdot \mathbf{L}^{-\mathbf{1}}$ & Standard Deviation $/ \mathbf{g} \cdot \mathbf{L}^{-\mathbf{1}}$ & $\mathbf{M a x} / \mathbf{g} \cdot \mathbf{L}^{-\mathbf{1}}$ & $\mathbf{M i n} / \mathbf{g} \cdot \mathbf{L}^{-\mathbf{1}}$ \\
\hline DAD & 0.0015 & 0.020 & 0.066 & -0.023 \\
Fluorescence & 0.0080 & 0.006 & 0.020 & -0.003 \\
Combination & 0.0003 & 0.018 & 0.025 & -0.029 \\
FTIR & 0.1266 & 0.061 & 0.204 & 0.056 \\
\hline
\end{tabular}

\section{Simulation Results of Advanced Process Control in iCCC through PAT}

This simulation study aims to demonstrate the efficiency of using online measurement data in process control of chromatographic processes. Furthermore, it aims to demonstrate that the achieved accuracy is high enough to implement a reliable process control. While process control and process disturbances can be handled with a simple process control, which will be shown in this part, a process online optimization has to be employed using model predictive Controllers (MPC). MPC are one of the most utilized methods of advanced process control (APC) [36] and enable automated process optimization utilizing optimization routines [36-38]. The main problem of MPC-based APC is process drift due to aging, fouling or blocking [38-40]. This drift can be implemented in the simulation using the online data obtained with the PAT methods described in this paper, enabling a real-time feedback of the process and allow for a continuous parameter fit [39,40].

\subsection{Parameter Estimation}

The results of parameter estimation are shown in Figure 13 Results of parameter estimation (a) is monomer, (b) dimer, (c) LMW 1 and (d) LMW 2. Simulation results are given as lines, fractions are given as dashes.

Correlation of component concentrations were considered acceptable. It should be emphasized that the main goal of this simulation is the demonstration of the possible process control. For process design a more sophisticated parameter determination method should be used, as previously described by Zobel-Roos et al. [10]. This would enable a better fit, as the amount of correlated parameters decrease, e.g., $\mathrm{D}_{\mathrm{ax}}, \mathrm{D}_{\mathrm{p}, \mathrm{i}}$, and $\mathrm{k}_{\mathrm{f}, \mathrm{i}}$. 


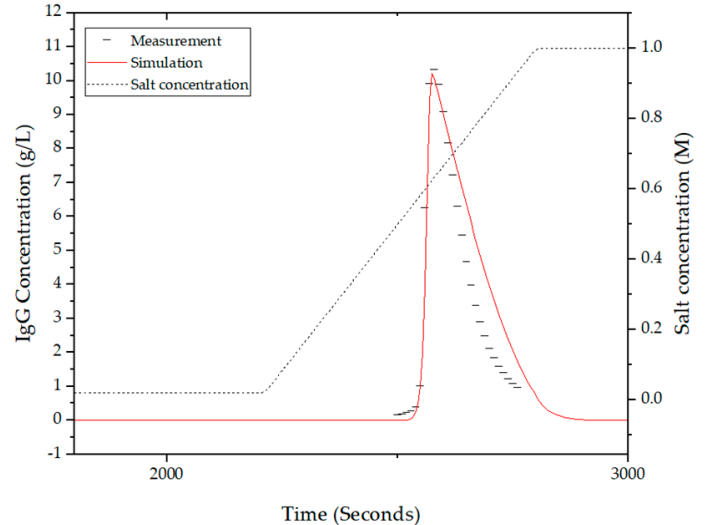

(a)

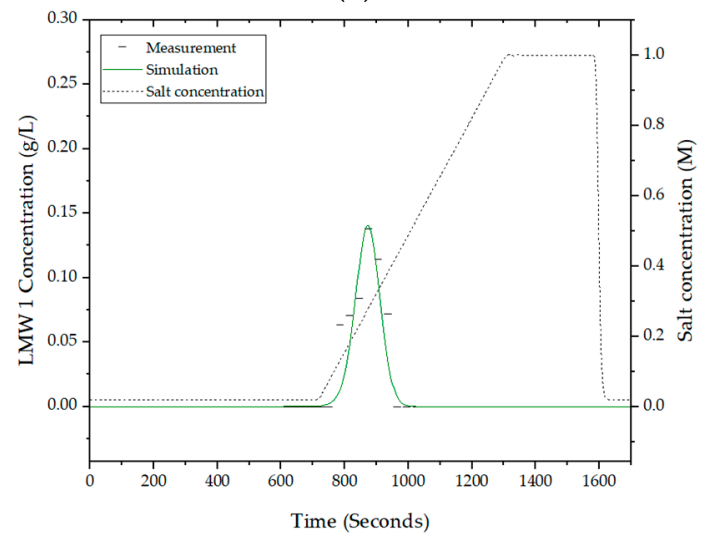

(c)

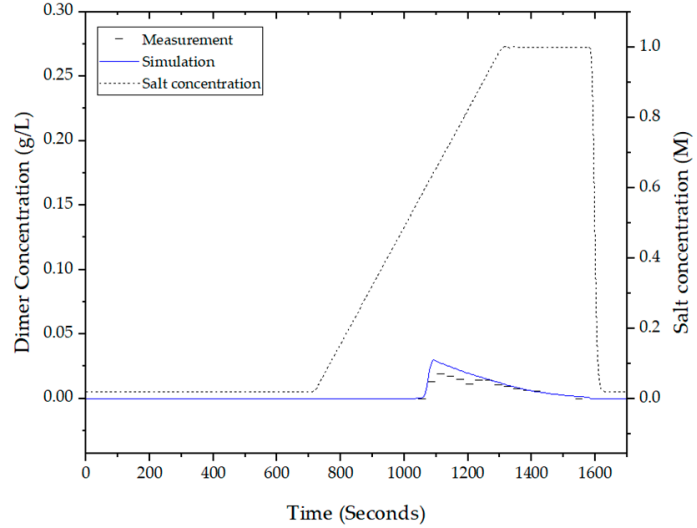

(b)

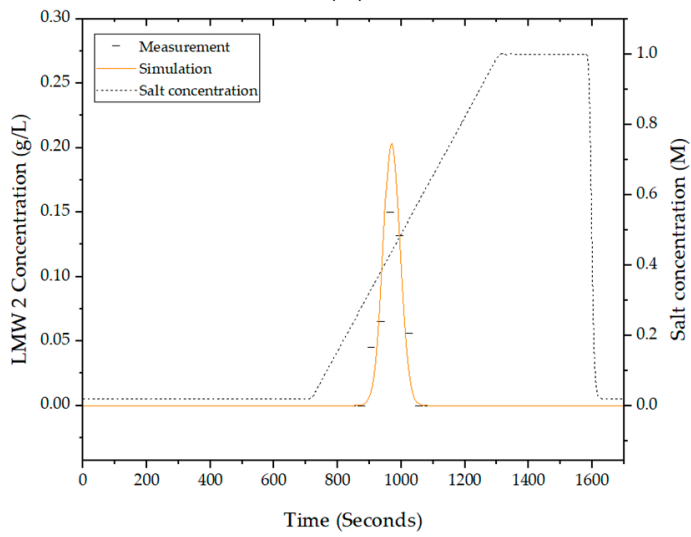

(d)

Figure 13. Results of parameter estimation (a) is monomer, (b) dimer, (c) LMW 1 and (d) LMW 2. Simulation results are given as lines, fractions are given as dashes.

\section{2. iCCC Modelling}

In Figure 14 simulation results from iCCC modelling are illustrated. In (a) the chromatogram of IEX is given. Cutting points are marked with dashed lines. For fractionation the PAT array is simulated, detecting main and side components. Based on the monitored concentrations the fractions are cut. Using this strategy, a high purity of over $99.9 \%$ can be achieved, while maintaining a high yield.

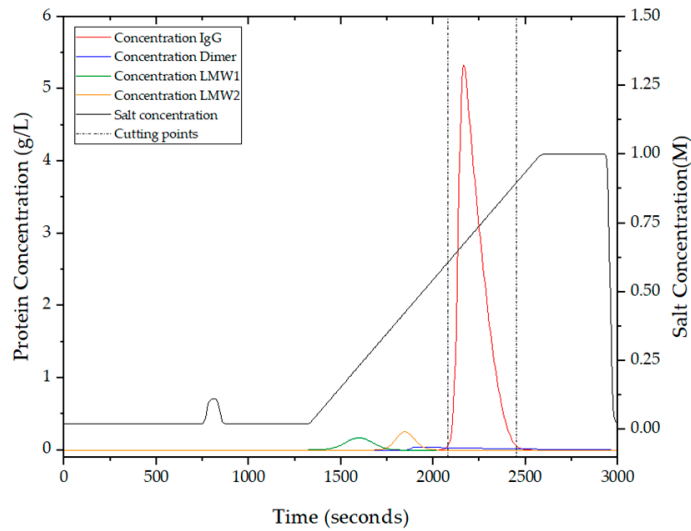

(a)

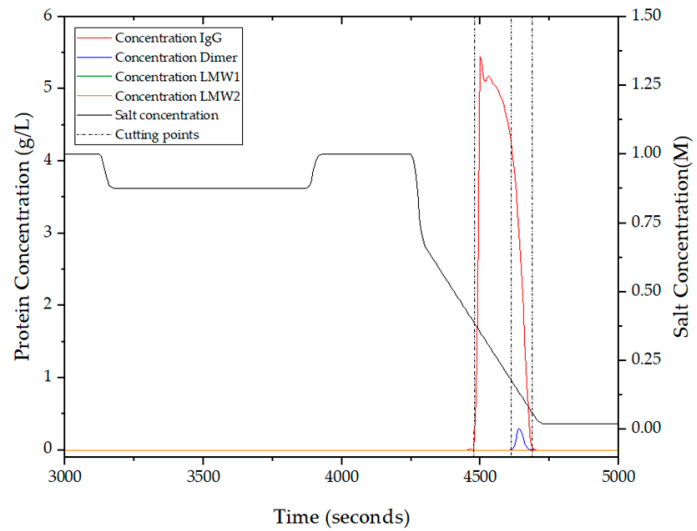

(b)

Figure 14. Simulation results of iCCC. (a) is IEX after five cycles, (b) is HIC after five cycles. 
Using this simulation of process control, different disturbances can be simulated for iCCC. To simulated process disturbances, feed concentration after precipitation and dissolution is varied from 0.5 to $1.5 \mathrm{~g} / \mathrm{L}$, flow rate is varied from 0.5 to $1.5 \mathrm{~mL} / \mathrm{min}$ and feed purity is varied from 85 to $95 \%$. This is done by varying the existing side component concentration in the feed by $\pm 50 \%$. In Table 4 the results of these runs are given. The yield in cycle 5 is between 90 and 99\%. As the process holdup between the cycles is built up, the step yield is steadily increasing. This takes more time, if there is less antibody fed into the process per step, explaining the lower overall yield for low concentration and process flowrate. The step yield increases until it reaches the endpoint of $99 \%$. The product is lost, as the elution of the IgG starts until its concentration reaches $0.05 \mathrm{~g} / \mathrm{L}$ and after it falls below $0.05 \mathrm{~g} / \mathrm{L}$.

Table 4. Simulation results of iCCC with process disturbances after five cycles.

\begin{tabular}{cccc}
\hline Run & Yield Cycle 5/\% & Concentration Cycle 5/g. $\mathbf{L}^{-\mathbf{1}}$ & Overall Yield/\% \\
\hline Standard & 97.0 & 4.4 & 87.5 \\
High IgG concentration & 97.9 & 4.7 & 91.1 \\
High process flowrate & 98.2 & 4.9 & 93.0 \\
High Purity & 95.5 & 4.4 & 89.3 \\
Low IgG concentration & 90.3 & 3.8 & 78.4 \\
Low process flowrate & 90.6 & 3.8 & 78.6 \\
Low Purity & 97.0 & 4.4 & 87.4 \\
\hline
\end{tabular}

Considering the concentrations, it is apparent that the process flow rate, and feed concentration has an influence on product concentrations. This is due to the changed product mass loaded on the column. Higher concentration and lower process volume or vice versa often appear in conjunction. These effects compensate each other, if controlled by a PAT-aided process control. This is shown in Table 5. To adjust process time a higher or lower flow rate can be used. This results in a flow rate of $0.875 \mathrm{~mL} / \mathrm{min}$ for the lower process volume, and $1.20 \mathrm{~mL} / \mathrm{min}$ for the higher volume. This adjustment of column flow rate is not commonly used in chromatography, since it may alter the process result, and retention times, resulting in a lower process yield. This is due to the changed height of the theoretical plates, according to van Deemter. The risk of missing the target component fraction can be eliminated though with an in-line concentration measurement, as the cutting points are automatically adjusted.

Table 5. Simulation results of iCCC for high and low feed concentrations, adjusting loading volume.

\begin{tabular}{cccc}
\hline Run & Yield Cycle 5/\% & Concentration Cycle 5/g. $\mathbf{L}^{-\mathbf{1}}$ & Overall Yield/\% \\
\hline $\begin{array}{c}\text { High IgG concentration, } \\
\text { low process flow rate }\end{array}$ & 95.1 & 4.2 & 83.3 \\
$\begin{array}{c}\text { Low IgG concentration, } \\
\text { high process flow rate }\end{array}$ & 96.9 & 4.4 & 87.4 \\
\hline
\end{tabular}

In Figure 15 the chromatograms for the iCCC start-up are given. As the IgG load on the column increases, due to the low-salt fractions from IEX and HIC loaded on the column, the elution time of the product changes. This change in elution time can be detected using the above described PAT system, avoiding product loss, and simplifying process start-up. 


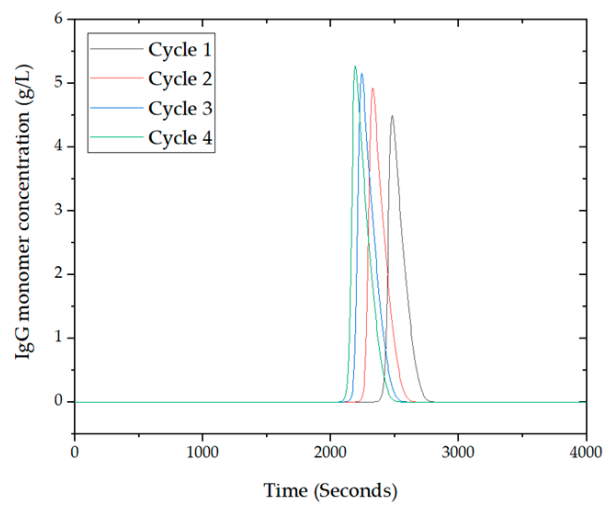

Figure 15. IEX chromatograms of iCCC start-up.

\subsection{Process Reaction to Disturbances}

In Figure 16 the course of the process after one cycle with lower IgG concentration in the feed is shown, (a) is with timed control of fraction cuts (b) is with automatic control based on concentration data from PAT. In this case, a low concentration cycle takes place in cycle 11. Due to the lower concentration the elution time of the product changes and fraction cuts have to be adjusted. This is enabled by real-time concentration measurement, realizable using the methods described above. Employing this process control, yield and concentration in the following cycles is controlled and the need for manual input is eliminated. Furthermore, the smaller variance in product concentration significantly lowers concentration and formulation efforts.

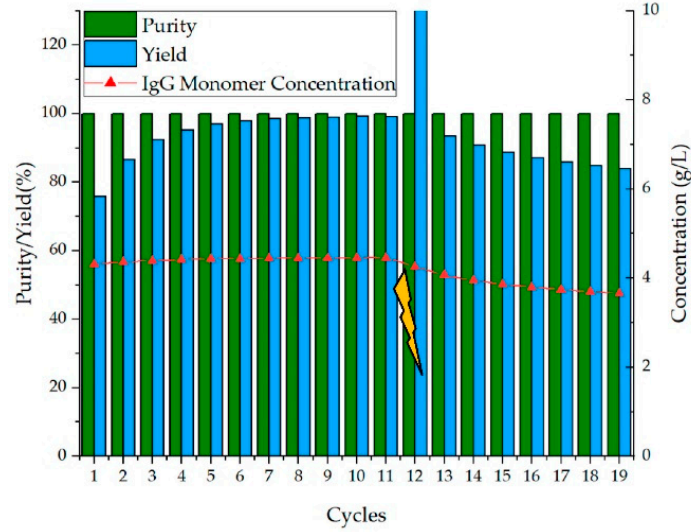

(a)

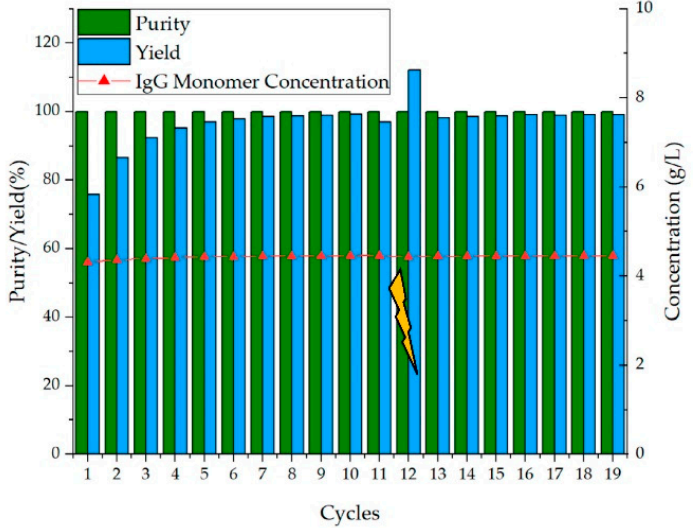

(b)

Figure 16. Purity, Yield and concentration course over cycles. (a) is without process control, (b) is with valve control via PAT concentration measurement. Concentration disturbance occurs in cycle 11.

In graph (a) and (b) it is apparent that after the process disturbance in cycle 11 the step yield of the following step increases to over $100 \%$. This is a result of the lower product mass injected, while the tanks installed in the iCCC process still hold the product from the previous step. In (a) the process drifts away from the optimal continuous production, while in (b) process control efficiently handles the disturbance. Average yield after the disturbance is $87.8 \%$ for (a) and $98.9 \%$ for (b). This corresponds to a yield increase of $11.1 \%$.

Process control via fractionation based on concentration measurement is equally successful if the feed volume is decreased for one cycle, which is realistic for this operation as the last step in $\mathrm{mAb}$ manufacturing. Here, pump flow was decreased to stay in the same process tact. Figure 17 depicts the results of this simulation. In (a) the process again drifts away from the optimal continuous production, while (b) handles the disturbance successfully. Average yield after the disturbance is $86.3 \%$ in (a) and $98.8 \%$ in (b), corresponding to a yield increase of $12.5 \%$. 


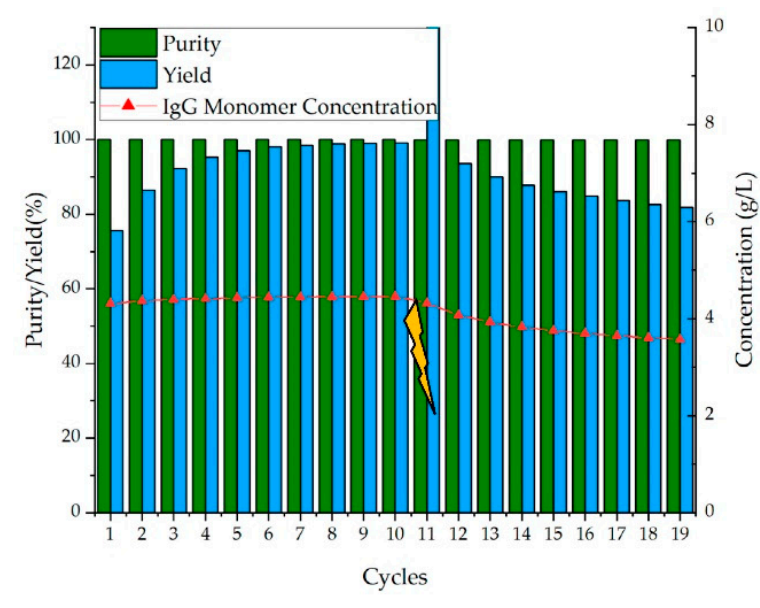

(a)

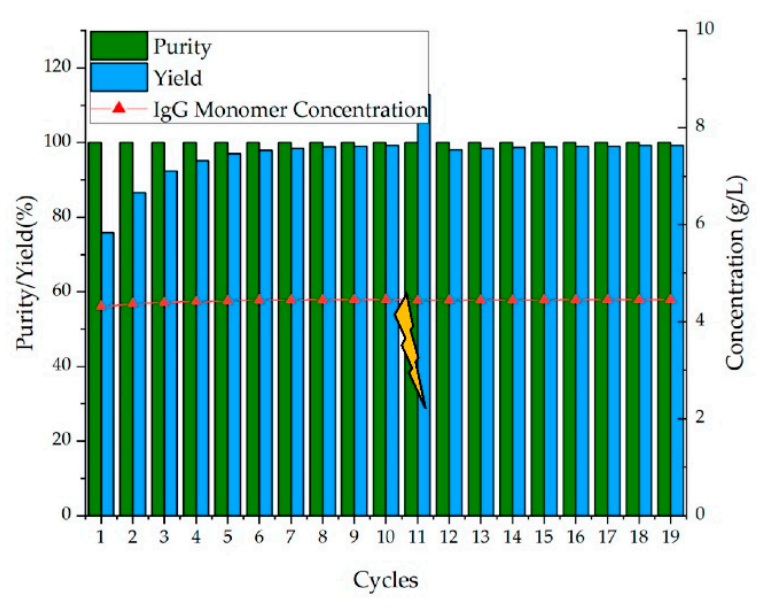

(b)

Figure 17. Purity, yield, and concentration course over cycles. (a) is without process control, (b) is with valve control via PAT concentration measurement. Volume disturbance occurs in cycle 11.

This simulation shows that an in-line process control using a PAT system of DAD and/or fluorescence lead to a very high process yield, since cutting points in the chromatogram can be automatically detected, controlling the fractionation. Using this system for process control can efficiently handle large process disturbances and parameter fluctuations of up to $50 \%$.

\section{Discussion}

Advanced process control using inline measurements and chemometrics is the most promising way to establish robust continuous downstream manufacturing in the purification of biopharmaceuticals. One of the main reasons for inline spectroscopic data not being widely used for process control is the lacking specificity for a target molecule if other components are present. This problem can be eliminated when a combination of different spectroscopic methods is used. The combination of DAD and fluorescence spectra, eliminated the high variation in zero-point fluctuations observed in DAD while simultaneously enabling the measurement of the main and side components.

FTIR is possible to use in the observed experiments. However, it has the downside of having a lower sensitivity, detecting incoming peaks later and therefore would result in a lower process yield in preparative process. The lower sensitivity is observable in the zero-concentration samples, where up to $0.2 \mathrm{~g} / \mathrm{L}$ were calculated in samples, which do not contain IgG. This lower sensitivity observed in chromatography probably results in the overlapping absorbance ranges of the changing buffer solution and the target component, discussed above. Nevertheless, FTIR is a promising way to detect the secondary structure of proteins, but since no denaturation of IgG was observed in ELISA using this process, an evaluation was not possible. Raman spectroscopy was not employed successfully. In literature this was possible using a smaller flow-cell for a breakthrough chromatography process.

In Figure 18, a control strategy for iCCC is proposed. To calculate the required loading time, the feed concentration is measured using FTIR or DAD as described in the overview paper of this study [24] in front of the feed tank (blue). Data used for release testing is obtained by measurement arrays located after the columns (green). Based on the results shown in this paper, the most reliable detection method for IgG monomer and the side components is a combination of fluorescence and UV-VIS spectroscopy. Coupling these spectroscopic methods with a PLS model allows for a real-time online concentration measurement. 


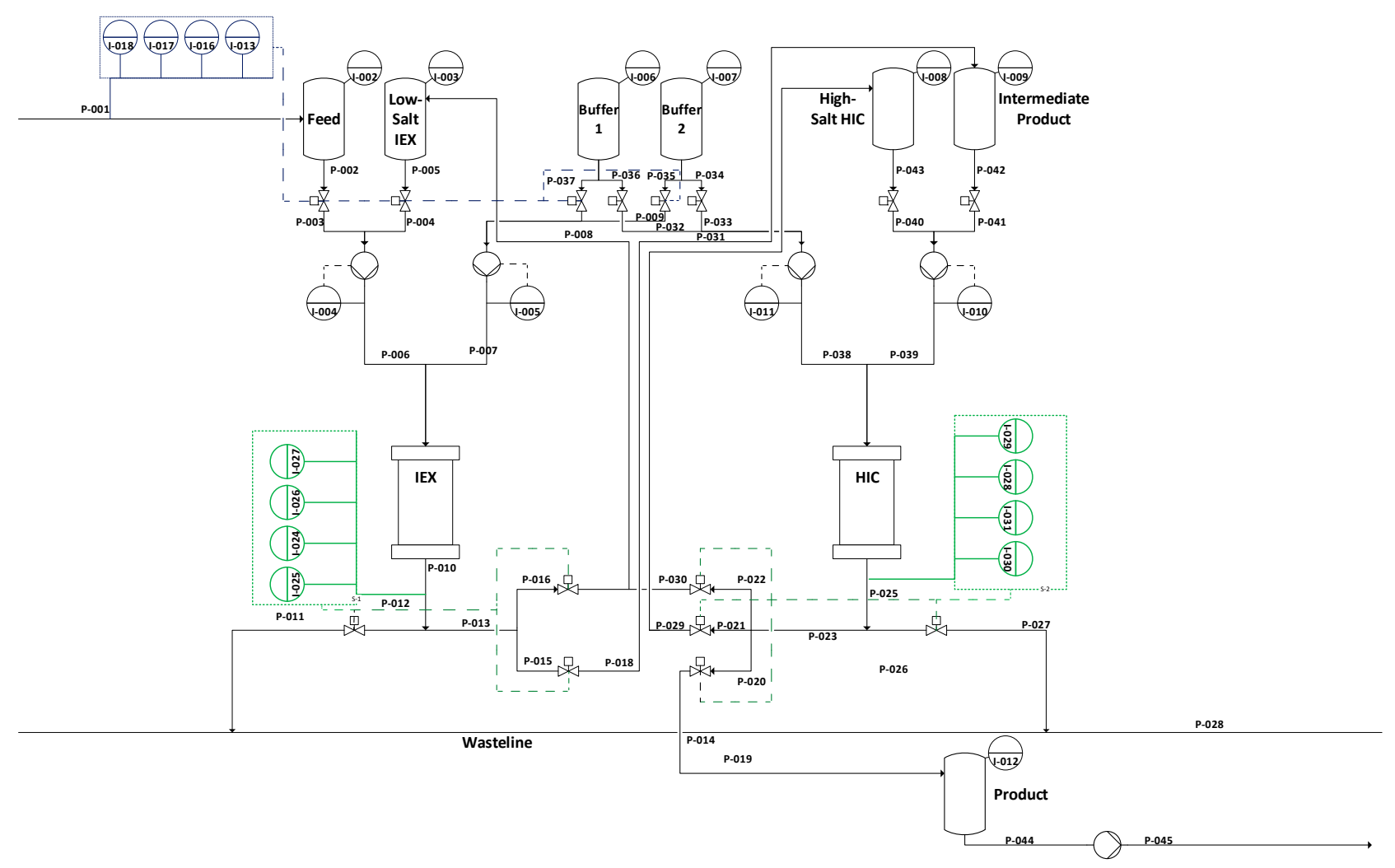

Figure 18. Proposed control strategy for iCCC.

Based on these measurements two different control strategies can be used. Employing a digital twin, i.e., a process model predicting process results parallel the physical process, would allow for fractionation control, process optimization, and the monitoring of ageing processes. The elution times of the main and side components can be calculated with which the fractionation valves can be controlled. The other control strategy is the fractionation control using the inline concentration measurements, which is modelled in this work. The described control system is able to regulate process disturbances of up to $50 \%$ variance on the feed concentration and volume, while maintaining a constant concentration and process yield. Compared to a timed fraction cut the yield could be increased by up to $12.5 \%$. In addition to iCCC this detection method can also be used for multicolumn counter-current solvent gradient purification (MCSGP) or regular batch chromatography.

In addition to spectroscopic data, other sensor data, such as conductivity, $\mathrm{pH}$ or osmolarity have shown small improvements to the regression of the target component [41] but did not show significant impact to regression quality in this work [24]. From our viewpoint the prediction of osmolarity using spectroscopic data, conductivity and $\mathrm{pH}$ appear to be promising. Osmolarity is a critical quality attribute (CQA) for pharmaceuticals. A real-time measurement of this CQA with sensors already employed in chromatography (e.g., UV-VIS, $\mathrm{pH}$, and conductivity) would reduce the analytical effort and further realtime release testing (RTRT).

As a part of a RTRT as proposed by regulatory authorities, inline concentration and purity measurement will play a big role. Other CQA as biological efficiency or glycosylation were reportedly possible to control using FTIR [18-20,42]. Additionally, controlling these parameters with spectroscopy, semi-automation of process analytics are becoming more viable as an important addition to inline data producing data about biological efficiency via ELISA in an acceptable timeframe [43]. 


\section{Conclusions}

In this study a reliable inline PAT concept for the evaluation of chromatography product stream has been developed. By combining DAD and fluorescence measurement a steadier zero-point detection is possible, allowing earlier and more reliable measurement of different chromatography fractions. For component measurements a $\mathrm{R}^{2}$ of 0.93 for the target component was reached. The RMSE was 0.027 for the target component. For the dimer a $R^{2}$ of 0.67 was reached with the combined model and a $R^{2}$ of 0.84 was reached with fluorescence measurements only. For the side components identified to be both light molecular weight components a $R^{2}$ of 0.91 for component 1 and a $R^{2}$ of 0.93 for component 2 was reached. Allowing a measurement of all observed side components with the same PLS model. Based on this PLS-model a simulation for the iCCC process was conducted, using the detection limit observed in the experiments. This led to a consistent cycle yield in iCCC of $>99.9 \%$ even with different process disturbances. To demonstrate the reliability of this process control concept variations of $\pm 50 \%$ for processed feed volume, feed purity and feed IgG concentration were simulated. Compared to a timed process control, yield is increased by up to $12.5 \%$, if unexpected process disturbances occur.

Author Contributions: Conceptualization: J.S.; methodology, experimental design and evaluation: F.L.V.; writing, editing, and reviewing: F.L.V., S.Z.-R. and J.S.; supervision: J.S. All authors have read and agreed to the published version of the manuscript.

Funding: The authors want to gratefully acknowledge the Bundesministerium für Wirtschaft und Energie (BMWi), especially Michael Gahr (Projektträger FZ Jülich), for funding the scientific work. We also kindly acknowledge the support by Open Access Publishing Fund of Clausthal University of Technology.

Institutional Review Board Statement: Not applicable.

Informed Consent Statement: Not applicable.

Data Availability Statement: Data cannot be made publicly available.

Acknowledgments: The authors would like to thank Reinhard Ditz, formerly of Merck KGaA, Darmstadt, for paper revision and fruitful discussions, as well as the ITVP lab-team, especially Frank Steinhäuser, Volker Strohmeyer and Thomas Knebel, for their efforts and support.

Conflicts of Interest: The authors declare no conflict of interest. The funders had no role in the design of the study; in the collection, analyses, or interpretation of data; in the writing of the manuscript, or in the decision to publish the results.

\section{References}

1. Kornecki, M.; Schmidt, A.; Lohmann, L.; Huter, M.; Mestmäcker, F.; Klepzig, L.; Mouellef, M.; Zobel-Roos, S.; Strube, J. Accelerating Biomanufacturing by Modeling of Continuous Bioprocessing-Piloting Case Study of Monoclonal Antibody Manufacturing. Processes 2019, 7, 495. [CrossRef]

2. Feidl, F.; Vogg, S.; Wolf, M.; Podobnik, M.; Ruggeri, C.; Ulmer, N.; Wälchli, R.; Souquet, J.; Broly, H.; Butté, A.; et al. Process-wide control and automation of an integrated continuous manufacturing platform for antibodies. Biotechnol. Bioeng. 2020, 117, 1367-1380. [CrossRef]

3. Otto, R.; Santagostino, A.; Schrader, U. Rapid Growth in Biopharma: Challenges and Opportunities. 2014. Available online: https: / / www.mckinsey.com.br/ \{\}/media/McKinsey/Industries/Healthcare\%20Systems\%20and\%20Services/Our\%20Insights / Rapid\%20growth\%20in\%20biopharma/Rapid\%20growth\%20in\%20biopharma\%20Challenges\%20and\%20opportunities.pdf (accessed on 6 March 2021).

4. Walther, J.; Godawat, R.; Hwang, C.; Abe, Y.; Sinclair, A.; Konstantinov, K. The business impact of an integrated continuous biomanufacturing platform for recombinant protein production. J. Biotechnol. 2015, 213, 3-12. [CrossRef]

5. Kornecki, M.; Strube, J. Accelerating Biologics Manufacturing by Upstream Process Modelling. Processes 2019, 7, 166. [CrossRef]

6. Subramanian, G. Continuous Biomanufacturing_Innovative Technologies and Methods; Wiley-VCH: Weinheim, Germany, 2017; ISBN 9783527699902.

7. Kamga, M.-H.; Cattaneo, M.; Yoon, S. Integrated continuous biomanufacturing platform with ATF perfusion and one column chromatography operation for optimum resin utilization and productivity. Prep. Biochem. Biotechnol. 2018, 48, 383-390. [CrossRef]

8. Vogg, S.; Müller-Späth, T.; Morbidelli, M. Design space and robustness analysis of batch and counter-current frontal chromatography processes for the removal of antibody aggregates. J. Chromatogr. A 2020, 1619, 460943. [CrossRef] 
9. Zobel-Roos, S.; Schmidt, A.; Mestmäcker, F.; Mouellef, M.; Huter, M.; Uhlenbrock, L.; Kornecki, M.; Lohmann, L.; Ditz, R.; Strube, J. Accelerating Biologics Manufacturing by Modeling or: Is Approval under the QbD and PAT Approaches Demanded by Authorities Acceptable Without a Digital-Twin? Processes 2019, 7, 94. [CrossRef]

10. Zobel-Roos, S.; Mouellef, M.; Ditz, R.; Strube, J. Distinct and Quantitative Validation Method for Predictive Process Modelling in Preparative Chromatography of Synthetic and Bio-Based Feed Mixtures Following a Quality-by-Design (QbD) Approach Processes 2019, 7, 580. [CrossRef]

11. Zobel-Roos, S.; Mouellef, M.; Siemers, C.; Strube, J. Process Analytical Approach towards Quality Controlled Process Automation for the Downstream of Protein Mixtures by Inline Concentration Measurements Based on Ultraviolet/Visible Light (UV/VIS) Spectral Analysis. Antibodies 2017, 6, 24. [CrossRef]

12. Godawat, R.; Brower, K.; Jain, S.; Konstantinov, K.; Riske, F.; Warikoo, V. Periodic counter-current chromatography-Design and operational considerations for integrated and continuous purification of proteins. Biotechnol. J. 2012, 7, 1496-1508. [CrossRef]

13. Müller-Späth, T.; Aumann, L.; Melter, L.; Ströhlein, G.; Morbidelli, M. Chromatographic separation of three monoclonal antibody variants using multicolumn countercurrent solvent gradient purification (MCSGP). Biotechnol. Bioeng. 2008, 100, $1166-1177$. [CrossRef] [PubMed]

14. Strube, J. Technische Chromatographie: Auslegung, Optimierung, Betrieb und Wirtschaftlichkeit; Shaker: Aachen, Germany, 2000; ISBN 3826568974.

15. Strube, J.; Klatt, K.-U.; Noeth, G.; Greifenberg, J.; Boecker, S.; Kansy, H.; Jaehn, P.; Justen, B. Method for the Production of Chemical and Pharmaceutical Products with Integrated Multicolumn Chromatography. U.S. Patent 7,615,159, 10 November 2009.

16. Brestrich, N.; Rüdt, M.; Büchler, D.; Hubbuch, J. Selective protein quantification for preparative chromatography using variable pathlength UV/Vis spectroscopy and partial least squares regression. Chem. Eng. Sci. 2018, 176, 157-164. [CrossRef]

17. Feidl, F.; Garbellini, S.; Vogg, S.; Sokolov, M.; Souquet, J.; Broly, H.; Butté, A.; Morbidelli, M. A new flow cell and chemometric protocol for implementing in-line Raman spectroscopy in chromatography. Biotechnol. Prog. 2019, 35, e2847. [CrossRef]

18. Wang, Y.; Boysen, R.I.; Wood, B.R.; Kansiz, M.; McNaughton, D.; Hearn, M.T.W. Determination of the secondary structure of proteins in different environments by FTIR-ATR spectroscopy and PLS regression. Biopolymers 2008, 89, 895-905. [CrossRef] [PubMed]

19. Boulet-Audet, M.; Kazarian, S.G.; Byrne, B. In-column ATR-FTIR spectroscopy to monitor affinity chromatography purification of monoclonal antibodies. Sci. Rep. 2016, 6, 30526. [CrossRef]

20. Walther, C.; Mayer, S.; Jungbauer, A.; Dürauer, A. Getting ready for PAT: Scale up and inline monitoring of protein refolding of Npro fusion proteins. Process. Biochem. 2014, 49, 1113-1121. [CrossRef]

21. Sauer, D.G.; Melcher, M.; Mosor, M.; Walch, N.; Berkemeyer, M.; Scharl-Hirsch, T.; Leisch, F.; Jungbauer, A.; Dürauer, A. Real-time monitoring and model-based prediction of purity and quantity during a chromatographic capture of fibroblast growth factor 2. Biotechnol. Bioeng. 2019, 116, 1999-2009. [CrossRef]

22. Rolinger, L.; Rüdt, M.; Diehm, J.; Chow-Hubbertz, J.; Heitmann, M.; Schleper, S.; Hubbuch, J. Multi-attribute PAT for UF/DF of Proteins-Monitoring Concentration, particle sizes, and Buffer Exchange. Anal. Bioanal. Chem. 2020, 412, 2123-2136. [CrossRef] [PubMed]

23. Zobel, S.; Helling, C.; Ditz, R.; Strube, J. Design and Operation of Continuous Countercurrent Chromatography in Biotechnological Production. Ind. Eng. Chem. Res. 2014, 53, 9169-9185. [CrossRef]

24. Helgers, H.; Schmidt, A.; Lohmann, L.J.; Vetter, F.L.; Juckers, A.; Jensch, C.; Mouellef, M.; Zobel-Roos, S.; Strube, J. Towards Autonomous Operation by Advanced Process Control-Process Analytical Technology for Continuous Biologics Antibody Manufacturing. Processes 2021, 9, 172. [CrossRef]

25. Guiochon, G. Fundamentals of Preparative and Nonlinear Chromatography, 2nd ed.; Elsevier: Amsterdam, The Netherlands, 2006; ISBN 9780123705372.

26. Zobel-Roos, S. Entwicklung, Modellierung und Validierung von Integrierten Kontinuierlichen Gegenstrom-ChromatographieProzessen. Ph.D. Thesis, Technische Universität Clausthal, Clausthal, Germany, 2018.

27. Dünnebier, G.; Engell, S.; Epping, A.; Hanisch, F.; Jupke, A.; Klatt, K.-U.; Schmidt-Traub, H. Model-based control of batch chromatography. AIChE J. 2001, 47, 2493-2502. [CrossRef]

28. Brooks, C.A.; Cramer, S.M. Steric mass-action ion exchange: Displacement profiles and induced salt gradients. AIChE J. 1992, 38, 1969-1978. [CrossRef]

29. Carta, G.; Jungbauer, A. Protein chromatography. In Process Development and Scale-Up; Wiley-VCH: Weinheim, Germany, 2010; ISBN 978-3-527-31819-3.

30. Seidel-Morgenstern, A.; Guiochon, G. Modelling of the competitive isotherms and the chromatographic separation of two enantiomers. Chem. Eng. Sci. 1993, 48, 2787-2797. [CrossRef]

31. Langmuir, I. The adsorption of gases on plane surfaces of glass, mica and platinum. J. Am. Chem. Soc. 1918, 40, 1361-1403. [CrossRef]

32. Leśko, M.; Åsberg, D.; Enmark, M.; Samuelsson, J.; Fornstedt, T.; Kaczmarski, K. Choice of Model for Estimation of Adsorption Isotherm Parameters in Gradient Elution Preparative Liquid Chromatography. Chromatographia 2015, 78, 1293-1297. [CrossRef]

33. Seidel-Morgenstern, A. Experimental determination of single solute and competitive adsorption isotherms. J. Chromatogr. A 2004, 1037, 255-272. [CrossRef] 
34. Carta, G.; Rodrigues, A.E. Diffusion and convection in chromatographic processes using permeable supports with a bidisperse pore structure. Chem. Eng. Sci. 1993, 48, 3927-3935. [CrossRef]

35. Wilson, E.J.; Geankoplis, C.J. Liquid Mass Transfer at Very Low Reynolds Numbers in Packed Beds. Ind. Eng. Chem. Fundam. 1966, 5, 9-14. [CrossRef]

36. Dittmar, R. Advanced Process Control: PID-Basisregelungen, Vermaschte Regelungsstrukturen, Softsensoren, Model Predictive Control; De Gruyter: Berlin, Germany, 2017; ISBN 978-3-11-049997-1.

37. Keerthi, S.S.; Gilbert, E.G. Optimal infinite-horizon feedback laws for a general class of constrained discrete-time systems: Stability and moving-horizon approximations. J. Optim. Theory Appl. 1988, 57, 265-293. [CrossRef]

38. Song, I.-H.; Amanullah, M.; Erdem, G.; Mazzotti, M.; Rhee, H.-K. Experimental implementation of identification-based optimizing control of a simulated moving bed process. J. Chromatogr. A 2006, 1113, 60-73. [CrossRef] [PubMed]

39. Sommeregger, W.; Sissolak, B.; Kandra, K.; Von Stosch, M.; Mayer, M.; Striedner, G. Quality by control: Towards model predictive control of mammalian cell culture bioprocesses. Biotechnol. J. 2017, 12. [CrossRef]

40. Fanali, S. Editorial on "Simulated moving bed chromatography for the separation of enantiomers" by A. Rajendran, G. Paredes and M. Mazzotti. J. Chromatogr. A 2009, 1216, 708. [CrossRef]

41. Felfödi, E.; Scharl, T.; Melcher, M.; Dürauer, A.; Wright, K.; Jungbauer, A. Osmolality is a predictor for model-based real time monitoring of concentration in protein chromatography. J. Chem. Technol. Biotechnol. 2019, 14, 1146-1152. [CrossRef]

42. Buijs, J.; Norde, W.; Lichtenbelt, J.W.T. Changes in the Secondary Structure of Adsorbed IgG and F(ab')2Studied by FTIR Spectroscopy. Langmuir 1996, 12, 1605-1613. [CrossRef]

43. Christler, A.; Felföldi, E.; Mosor, M.; Sauer, D.; Walch, N.; Dürauer, A.; Jungbauer, A. Semi-automation of process analytics reduces operator effect. Bioprocess Biosyst. Eng. 2019, 43, 753-764. [CrossRef] 